\title{
Role of Sepsis Screen Tests in Predicting Neonatal Septicemia
}

\author{
B.I. Vedavati* and B. Amrutha Kumari \\ Department of Microbiology, Mysore Medical College and Research Institute, Mysore, \\ Karnataka, India \\ *Corresponding author
}

\begin{tabular}{|c|c|}
\hline & A B S T R A C T \\
\hline $\begin{array}{l}\text { Ke y w or d s } \\
\text { Sepsis screen, } \\
\text { Neonatal } \\
\text { septicemia, CRP, } \\
\text { I/T ratio, TLC, } \\
\text { ANC }\end{array}$ & $\begin{array}{l}\text { Early diagnosis of neonatal sepsis continues to pose a problem to the clinician caring for } \\
\text { newborns. Blood culture has been considered the gold standard for confirmation of } \\
\text { diagnosis. An array of sepsis screen tests are now available for early diagnosis of neonatal } \\
\text { sepsis. To study the role of sepsis screen tests in early diagnosis of neonatal sepsis. Blood } \\
\text { cultures and sepsis screen tests were carried out on } 200 \text { neonates. The identification of the } \\
\text { causative organism was carried out by standard identification tests. Sepsis screen was } \\
\text { carried out with C-reactive Protein, I/T ratio, Total leucocyte count, Absolute neutrophils }\end{array}$ \\
\hline Article Info & $\begin{array}{l}\text { count, Out of } 200 \text { neonates studied with clinical features of sepsis, } 128(64 \%) \text { were blood } \\
\text { culture positive. Among Sepsis screen tests, CRP was the most sensitive test }(89.84 \%)\end{array}$ \\
\hline $\begin{array}{l}\text { Accepted: } \\
\text { 24 January } 2018 \\
\text { Available Online: } \\
\text { 10 February } 2018\end{array}$ & $\begin{array}{l}\text { with a specificity of } 76.39 \% \text {. I/T ratio, TLC and ANC showed sensitivity } 76.56 \%, 44.53 \% \text {, } \\
32.81 \% \text { and specificity } 59.72 \% .76 .39 \%, 81.94 \% \text { respectively. Sepsis screen tests may help } \\
\text { and can be used as an adjunct in the diagnosis. They are also useful in early detection of } \\
\text { neonatal sepsis and can be used as prognostic indicators; thus helping to start early } \\
\text { treatment with appropriate antibiotics. }\end{array}$ \\
\hline
\end{tabular}

\section{Introduction}

Neonatal septicemia is a great masquerader and can present with very nonspecific manifestations pertaining to any system of the body (Jajoo et al., 2015). The incidence of neonatal sepsis in India is approximately 30/1000 live births (Misra et al., 2013). More than half of neonates admitted to neonatal intensive care units (NICUs) carry a diagnosis of "suspected sepsis" and these infants account for up to $25 \%$ of NICU days in some units (Jajoo et al., 2015). According to World Health Organization (WHO) estimates, there are about 5 million neonatal deaths a year, of which neonatal sepsis is the major cause. (Shrestha et al., 2007).

Early diagnosis of neonatal septicemia is a difficult task as the early signs of sepsis may be subtle, non-specific and different at different gestational ages and difficult to diagnose clinically (Aparna Narasimha and Harendra Kumar, 2011). There is no laboratory test with $100 \%$ sensitivity and specificity. Blood culture and sepsis screen are two most important diagnostic investigations. Blood culture has been considered the gold standard for confirmation of diagnosis but the results are available only after 48-72 hours. 
Moreover, in many cases blood culture fails to detect the causative organism (Mondal et al., 2012).

Neonatal sepsis is associated with the high mortality and serious morbidity so it is extremely important to make an early diagnosis of sepsis and prompt institution of empirical antimicrobial therapy. Hence, high index of suspicion is necessary for early diagnosis of sepsis. Test with a very high sensitivity and negative predictive value approaching $100 \%$ is desirable for diagnosis. However as no such tests are available; a battery of sepsis screen tests like CRP (Creactive protein), TLC (Total leucocyte count), ANC (Absolute Neutrophil count), I/T ratio (Immature to Total neutrophil ratio) and m-ESR (micro- erythrocyte sedimentation rate), may help in the diagnosis (Misra et al., 2013; Shatin, 2004). Studies have shown that septic screen tests are simple, quick, and cost- effective tools in the early diagnosis of neonatal sepsis. When these were studied together as combination of tests, it had proved that they increased both sensitivity and specificity. They are also useful early predictors of neonatal septicemia and also they are prognostic indicators; thus helping to initiate early treatment with appropriate antibiotics (Bhalodia et al., 2017). The present study was conducted to know the role of sepsis screen tests in the diagnosis of neonatal sepsis.

The main aim and objectives includes to study the role of sepsis screen tests in early diagnosis of neonatal sepsis.

\section{Materials and Methods}

\section{Source of Data}

The present study; "Role of sepsis screen tests in predicting neonatal septicemia" was carried out in Department of Microbiology, Mysore
Medical College \& Research Institute, Mysore over a period of one year from $1^{\text {st }}$ Jan $2014-$ $31^{\text {st }}$ Dec 2014.

\section{Method of collection of data}

Sample size: 200 clinically suspected cases of neonatal sepsis.

Inclusion criteria: Neonates admitted with signs and symptoms of sepsis.

Exclusion criteria: Neonates with no clinical evidence of sepsis.

\section{Collection of samples and processing}

After obtaining informed consent from parents/guardian, 1-5 ml blood was collected aseptically from neonates with suspected sepsis. 1-2 ml blood was inoculated into 5$10 \mathrm{ml}$ brain heart infusion broth and remaining blood into two different vials for different hematological and immunological tests. Broth was incubated at $37^{\circ} \mathrm{C}$ (Jajoo et al., 2015, Rajendraprasad et al., 2013). After 6-18 hours of incubation first subcultures were performed by aseptically removing a few drops of the well-mixed medium and inoculating onto blood agar, chocolate agar and MacConkey agar plate. The blood agar and MacConkey agar plates were incubated aerobically at $37^{\circ} \mathrm{C}$. Chocolate agar plate was incubated in 5 to $10 \%$ carbon dioxide jar at $35^{\circ} \mathrm{C}$ for 48 hours. Growth was further processed and identified according to standard protocols. If no growth was detected then further subculture were performed on $2^{\text {nd }}, 3^{\text {rd }}$ and $7^{\text {th }}$ day of incubation. Again growth was processed and identified as mentioned above (Forbes et al., 2014; Shrestha et al., 2007).

\section{Sepsis screen tests}

A battery of sepsis screen tests like CRP, TLC, Absolute Neutrophil Count, I/T ratio were carried out on the blood samples of all 
the neonates.

C-reactive protein was tested by Latex agglutination test using Bio-Scan kit. CRP $\geq$ $0.6 \mathrm{mg} / \mathrm{dl}$ was considered significant.

I/ $T$ ratio: It was calculated by examining peripheral blood smears stained with Giemsa stain. Blood smear was examined for immature neutrophils i.e., band forms, myelocytes and metamyelocytes. Immature neutrophils (band forms + myelocytes + metamyelocytes) to total neutrophil ratio were calculated. I/T neutrophil ratio greater than 0.2 was considered significant for neonatal sepsis

Total leucocyte count was estimated by using Sysmex automated analyser, which works on the principle of flow cytometry and counter verified by the pathologist. WBC count < $5000 / \mathrm{cmm}$ were considered positive for septicemia. Absolute Nutrophil count was also estimated similarly. A count $<1800 / \mathrm{cmm}$ were considered significant for sepsis (Kocabaş et al., 2007; Nayak et al., Misra et al., 2013).

\section{Results and Discussion}

The present study was carried out in the Department of Microbiology, Mysore Medical College and Research Institute, Mysore from January 2014 - December 2014. The observations made from the study are shown in tables 1-5.

Of 200 neonates studied, 126 were males, 74 were females and 128 (64\%) were blood culture positive.

Early diagnosis and treatment of neonatal sepsis is crucial for a favourable outcome. Blood culture is the gold standard in diagnosis of neonatal septicemia; which is positive in only $50-80 \%$ at best. A battery of sepsis screen tests may help and can be used as an adjunct in the diagnosis. Isolation of an organism from a sterile site is optimal for definitive diagnosis but it is not always possible to isolate a causative pathogen. Invasive infections can also occur in seemingly asymptomatic neonates (Shane and Stoll, 2014). In order to improve the outcome associated with neonatal sepsis, it is necessary for a diagnostic test to be rapid and sensitive to decrease delay in treatment. At the same time in order to avoid unnecessary exposure to antibiotics and invasive procedures, a test with higher specificity is needed. (Shah and Padbury, 2014) Sepsis screen tests may help in the diagnosis.

Sensitivity and specificity of CRP in our study is comparable with study conducted by Chauhan (Setal et al., 2012). Study conducted by Santhosh Kumar Mondal et al., reported $84 \%$ sensitivity and $65 \%$ specificity. (Mondal et al., 2012) Study conducted by Misra et al., showed $90.70 \%$ sensitivity and $37.50 \%$ specificity (Misra et al., 2013). CRP can be considered as a "specific" but "late" marker of neonatal infection.

Serial CRP measurements may also be helpful in monitoring the response to treatment in infected neonates and thus may help clinicians guide the duration of antibiotic therapy. If the CRP levels remain persistently normal, it correlates strongly with the absence of infection thereby guiding safe discontinuation of antibiotic therapy (Shah and Padbury, 2014).

I/T ratio $>0.2$ was considered as significant for neonatal sepsis. The maximum normal value is 0.16 during the first $24 \mathrm{hrs}, 0.14$ by 48 hours and 0.13 by 60 hours and it remains until 5 days of age. Thereafter the maximum normal $\mathrm{I} / \mathrm{T}$ ratio is 0.12 until the end of the first month. (Alison Bedford Russel David Isaacs) Ratio of $\geq 0.2$ suggests bacterial infection (Barbara and Andi). 
Table.1 Correlation of CRP with blood culture

\begin{tabular}{|c|c|c|c|c|c|c|}
\hline \multirow{2}{*}{ CRP } & \multicolumn{4}{|c|}{ BLOOD CULTURE } & \multirow{2}{*}{ TOTAL } \\
\cline { 2 - 5 } & \multicolumn{2}{|c|}{ POSITIVE } & \multicolumn{2}{c|}{ NEGATIVE } & \\
\hline Positive & $\mathbf{N}$ & \% & n & \% & \\
\hline Negative & 115 & 89.84 & 17 & 23.61 & 132 \\
\hline TOTAL & 13 & 10.15 & 55 & 76.38 & 68 \\
\hline & 128 & 100 & 72 & 100 & 200 \\
\hline
\end{tabular}

$\mathrm{CRP} \geq 0.6 \mathrm{mg} / \mathrm{dl}$ was taken as positive, among blood culture positive cases $115(89.84 \%)$ were CRP positive.

Table.2 Correlation of I/T ratio with blood culture

\begin{tabular}{|c|c|c|c|c|c|}
\hline \multirow{2}{*}{ J/T RATIO } & \multicolumn{4}{|c|}{ BLOOD CULTURE } & \multirow{2}{*}{ TOTAL } \\
\cline { 2 - 5 } & \multicolumn{2}{|c|}{ POSITIVE } & \multicolumn{1}{c|}{ NEGATIVE } & \\
\hline & $\mathbf{N}$ & $\mathbf{\%}$ & $\mathbf{n}$ & $\mathbf{\%}$ & \\
\hline$<0.2$ & 30 & 23.43 & 43 & 59.72 & 73 \\
\hline$>0.2$ & 98 & 76.56 & 29 & 40.27 & 127 \\
\hline TOTAL & 128 & 100 & 72 & 100 & 200 \\
\hline
\end{tabular}

Among blood culture positive cases, I/T ratio $>0.2$ is present in $98(76.56 \%)$ cases.

Table.3 Correlation of TLC with blood culture

\begin{tabular}{|c|c|c|c|c|c|}
\hline \multirow[t]{3}{*}{ TLC } & \multicolumn{4}{|c|}{ CULTURE } & \multirow[t]{3}{*}{ TOTAL } \\
\hline & \multicolumn{2}{|c|}{ POSITIVE } & \multicolumn{2}{|c|}{ NEGATIVE } & \\
\hline & n & $\%$ & $\mathbf{n}$ & $\%$ & \\
\hline$<5000$ & 57 & 44.53 & 17 & 23.61 & 74 \\
\hline $5000.01-20000$ & 63 & 49.21 & 52 & 72.22 & 115 \\
\hline$>20000$ & 8 & 6.25 & 3 & 4.16 & 11 \\
\hline \multirow[t]{2}{*}{ Total } & 128 & 100 & 72 & 100 & 200 \\
\hline & \multicolumn{5}{|c|}{$\mathrm{P}$ value $=0.007$} \\
\hline
\end{tabular}

In Blood culture positive cases, WBC count $<5000 / \mathrm{cmm}$ was present in $57(44.53 \%)$ cases.

Table.4 Correlation of ANC with blood culture

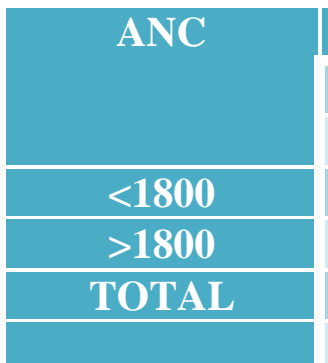

\begin{tabular}{|c|c|c|c|c|}
\hline \multicolumn{4}{|c|}{ BLOOD CULTURE } & \multirow[t]{3}{*}{ TOTAI } \\
\hline \multicolumn{2}{|c|}{ POSITIVE } & \multicolumn{2}{|c|}{ NEGATIVE } & \\
\hline $\mathbf{N}$ & $\%$ & $\mathbf{n}$ & $\%$ & \\
\hline 42 & 32.81 & 13 & 18.05 & 55 \\
\hline 86 & 67.18 & 59 & 81.94 & 145 \\
\hline 128 & 100 & 72 & 100 & 200 \\
\hline
\end{tabular}

In blood culture positive cases, Absolute neutrophil count (ANC) $<1800 / \mathrm{cmm}$ was present in $42(32.81 \%)$ cases. 
Table.5 Sensitivity and specificity of various sepsis screen tests in detecting Sepsis

\begin{tabular}{|c|c|c|c|c|}
\hline $\begin{array}{l}\text { SEPSIS SCREEN } \\
\text { TESTS }\end{array}$ & Sensitivity (\%) & Specificity (\%) & PPV (\%) & NPV (\%) \\
\hline CRP & 89.84 & 76.39 & 87.12 & 80.88 \\
\hline I/T ratio & 76.56 & 59.72 & 77.16 & 58.90 \\
\hline TLC & 44.53 & 76.39 & 77.02 & 43.65 \\
\hline ANC & 32.81 & 81.94 & 76.36 & 40.69 \\
\hline
\end{tabular}

However, the I: $\mathrm{T}$ ratio can be affected by various noninfectious processes like labor, prolonged induction with oxytocin, and even prolonged crying. Factors such as maternal hypertension or perinatal asphyxia may cause neutropenia or an elevated I/T ratio. Our study showed sensitivity of about $76.56 \%$ and specificity $59.72 \%$ with PPV $77.16 \%$ which is comparable with study conducted by Misra et al., Study by Arijit Majumdar et al., showed $100 \%$ sensitivity n $5 \%$ specificity (Majumdar et al., 2013). Study by Aparna Narasimha et al., showed $63.15 \%$ sensitivity and $75 \%$ specificity (Aparna Narasimha and Harendra Kumar, 2011). I/T ratio may be useful in diagnosing and monitoring infection.

A total leukocyte count of $<5000$ is significant for sepsis and can be used to infer the diagnosis of neonatal sepsis. Many infected newborns may have higher counts. Our study showed specificity of about $76 \%$ with high PPV which is comparable with study conducted by Aparna Narasimha et al., Study conducted Chauhan et al., Reported sensitivity of $30.77 \%$ and specificity of $63.15 \%$ (Setal et al., 2012). Reported sensitivity of low leukocyte count is only $29 \%$, but specificity can be as high as 91\%. Severe bacterial sepsis may also demonstrate an increase in TLC due to rise in mature and immature neutrophils. This could possibly be due to release of various growth factors and cytokines viz. G- CSF, GM- CSF, IL- 3, IL- 6; which stimulate bone marrow. Although CBC (complete blood count) has a poor predictive value, serial normal values can be used to enhance the prediction that bacterial sepsis is not present. However; leucopenia was a better predictor of septicemia as compared to leukocytosis as it has higher specificity (Shah and Padbury, 2014; Misra et al., 2013).

Absolute neutrophil count $<1800 / \mathrm{cmm}$ was considered significant for sepsis. In present study sensitivity, specificity, PPV, NPV were $32.81 \%, 81.94 \%, 76.36 \%, 40.69 \%$ respectively and it is comparable with study done by Misra et al., where in sensitivity, specificity, PPV is 20\%, 87.5\%, 75\% respectively (Misra et al., 2013). The ANC in babies born small for gestational age are lower than those born at weight appropriate for gestational age and may take up to 2 weeks of age to normalise (Alison Bedford Russel David Isaacs)

In summary sepsis screen should be done in all neonates suspected to have sepsis to corroborate the diagnosis. Among septic screen tests, WBC, $\mathrm{ANC}$ and $\mathrm{I} / \mathrm{T}$ ratio have significant limitations in the diagnosis of neonatal sepsis because of their low sensitivity. In our study CRP was the most sensitive test $(89.84 \%)$ with a specificity of $76.39 \%$. Sensitivity and specificity of the individual tests may not justify their individual use in newborn infants. Sepsis screen is considered positive if two of the tests are positive. If the screen is negative but clinical suspicion persists it should be repeated within 12 hours. Presence of two abnormal parameters in screen is associated with sensitivity 93$100 \%$, specificity $83 \%$, positive and negative predictive value of $27 \%$ and $100 \%$ respectively in detecting sepsis. ${ }^{19}$ Hence sepsis screen can be used as a predictor of sepsis to initiate therapy that can be modified based on blood culture reports. Early diagnosis of neonatal sepsis continues to pose a problem to the clinician caring for newborns. Battery of sepsis screen 
tests may help and can be used as an adjunct in the diagnosis. Sepsis screen tests are simple, quick, and cost- effective tools in the early diagnosis of neonatal sepsis and can be used as prognostic indicators; thus helping to start early treatment with appropriate antibiotics.

\section{References}

Alison Bedford Russel David Isaacs. Infection in the newborn, Rennie and Roberton's textbook of neonatology, London, $5^{\text {th }}$ ed. Elsevier. p; 1013-30.

Aparna Narasimha, and Harendra Kumar; Significance of hematological scoring system in early diagnosis of neonatal sepsis: Indian J Hematol Blood Transfus., 2011; 27(1):14-17

Barbara J stoll, and Andi L Shane. Infections of the neonatal infant. In: Nelson text book of paediatrics, $20^{\text {th }}$ ed. Elsevier. Volume 1, 909-25.

Bhalodia MJ, Hippargi SB, Patil MM. Role of hematological scoring system in diagnosis of neonatal sepsis. J ClinNeonatol., 2017; $6: 144-7$

Forbes, Sahm, Weissfeld. Bailey and Scott's Diagnostic Microbiology. $13^{\text {th }}$ edition China. 2014; chapter 68: 860 -877.

Jajoo M, Kapoor K, Garg LK, Manchanda V, Mittal SK. To study the incidence and risk factors of early onset neonatal sepsis in an out born neonatal intensive care unit of India. J Clin Neonatol., 2015; 4: 91-5.

Kocabaş E, Sarıkçıŏlu A, Aksaray N et al., Role of procalcitonin, C-reactive protein, interleukin-6, interleukin-8 and tumor necrosis factor- $\alpha$ in the diagnosis of neonatal sepsis. The Turkish Journal of Pediatrics 2007; 49: 7-20

Majumdar A, Jana A, Jana A, Biswas S, Bhattacharyya S. Hematologic scoring system (HSS): A guide to decide judicious use of antibiotics in neonatal septicemia in developing countries. J Appl Hematol 2013; 4: 110-3.

Misra RN, Jadhav SV, Ghosh P, Gandham N, Angadi K, Vyawahare C. Role of sepsis screen in the diagnosis of neonatal sepsis. Med J DY Patil Univ 2013; 6: 254-7.

Mondal SK, Nag DR, Bandyopadhyay R, Chakraborty D, Sinha SK. Neonatal sepsis: Role of a battery of immunohematological tests in early diagnosis. Int $\mathrm{J}$ App Basic Med Res., 2012; 2: 43-7

Mondal SK, Nag DR, Banyopadhyay R, Chakraborty D, Sinha SK. Neonatal sepsis Role of certain immunohematologic tests in rapid diagnosis. J Acad Med Sci., 2012; 2: 97-100.

Nayak R, Rai S, Gupta A. Cell count. Essentials in hematology and clinical pathology. New Delhi. 359 -85.

Rajendraprasad BM, Basavaraj KN, Antony B. Bacterial spectrum of neonatal septicemia with their antibiogram with reference to various predisposing factors in a tertiary care hospital in Southern India. Ann Trop Med Public Health 2013; 6: 96-9

Setal BC, Viren V, Bimal C. c-reactive protein (CRP) in early diagnosis of neonatal septicemia, national journal of medical research 2012; 2(3)P; 276-78

Shah BA, and Padbury JF. Neonatal sepsis, Virulence 2014.5: 1; 170-178

Shane AL, and Stoll BJ. Neonatal sepsis: Progress towards improved Outcomes. Journal of Infection 2014; 68: S24 -S32.

Shankarnarayan SC et al., Approach to neonatal sepsis. Jour- merine medical society 2015 , vol, 17. no $1 ; 12-19$

Shatin, NT. Diagnostic markers of infection in neonates. Arch Dis Child Fetal Neonatal Ed 2004; 89: F229-F235.

Shrestha P, Das BK, Bhatta NK et al., Clinical and Bacteriological profiles of blood culture positive sepsis in new borns. J Nepal Paediatr Soc 2007; 27(2): 64-7.

\section{How to cite this article:}

Vedavati, B.I. and Amrutha Kumari, B. 2018. Role of Sepsis Screen Tests in Predicting Neonatal Septicemia. Int.J.Curr.Microbiol.App.Sci. 7(02): 2522-2527. doi: https://doi.org/10.20546/ijcmas.2018.702.307 